\title{
How Plasmonic excitation influences the LIPSS formation on diamond during multipulse femtosecond laser irradiation?
}

\author{
Ahmed Abdelmalek ${ }^{1}$, Zeyneb Bedrane ${ }^{1, *}$,El-Hachemi Amara ${ }^{2}$, Shane M. Eaton ${ }^{3}$ and Roberta Ramponi ${ }^{3}$ \\ ${ }^{1}$ Theoretical Physics Laboratory, Physics Dpt. Sciences Faculty,Tlemcen University, B.P. 119, 13000 Tlemcen, Algeria \\ ${ }^{2}$ Laser Material Processing Team, Centre for Advanced Technologies Development, CDTA, PO Box 17 baba-Hassan, 16303 Algiers, \\ Algeria \\ ${ }^{3}$ Physics Dpt., Politecnico di Milano, Italy
}

\begin{abstract}
A generalized plasmonic model is proposed to calculate the nanostructure period induced by multipulse laser femtosecond on diamond at $800 \mathrm{~nm}$ wavelengths. We follow the evolution of LIPSS formation by changing diamond optical parameters in function of electron plasma excitation during laser irradiation. Our calculations shows that the ordered nanostructures can be observed only in the range of surface plasmon polariton excitation.
\end{abstract}

\section{INTRODUCTION}

Laser Induced Periodic Surface Structure, also called "ripples or nanograting" are part of morphologies that have focused the interest of several researchers and have been on the heart of a lot of studies since the first experiment done by Birnbaum in 1965 where two types of training LIPSS formation were observed during the irradiation of dielectric, semiconductor and metals [1, 2, 3] by multipulse femtosecond laser linearly polarized, called low spatial frequency LIPSS (LSFL) and high spatial frequency LIPSS (HSFL). LSFL formation for the majority of materials is well explained by the interference between the incident laser wave and the plasmon polaritons SPP surface [4, 3], which involves a periodic spatial modulation of the energy deposited in the irradiated surface $[5,6]$, this leads to the formation of nanostructures with periods lower or near to $\lambda_{\mathrm{L}}$.

But HSFL formation remain a misunderstood phenomenon and it is still under discussion. There are several models proposed and many studies were done to identify the origin of formation of this such type of LIPSS on dielectric and semiconductor, induced by multipulse laser femtosecond at low fluency [7, 3,
8, 9, 10]. In such cases, the nonthermal interaction and plasmon excitation play a very important role, where the HSFL's period is very lower to the incident laser wavelength. Masataka Shinoda et al [10] noted a period of $146 \pm 7 \mathrm{~nm}$ on diamond irradiated by $120 \mathrm{fs}, 250 \mathrm{kHz}$ at $\lambda_{\mathrm{L}}=800 \mathrm{~nm}$. Straub et al [11] find a period of $145 \mathrm{~nm}$ on the Si (100) at $\lambda_{\mathrm{L}}=800 \mathrm{~nm}$.

A recent explanation of HSFL formation was proposed by Miyazaki et al [9] and Miyaji et al [8] where they proposed that by increasing the number of low fluence pulses, the periodic nanostructures HSFL can be developed through the nonthermal structure change of the treated surface. The nanoscale ablation due to strong nearfields appears around of sweeling followed by a plasmonic excitation (SPP) to improve the nano-periodicity of irradiated area.

In this work, we illustate the plasmon excitation advantage to training HSFL formation through a general plasmon model that can follow the evolution of training LIPSS formation by changing diamond optical parameters in function of electron plasma excitation.

\footnotetext{
* Corresponding author: zeyneb_bedrane@yahoo.fr
} 


\section{THEORETICAL MODEL}

We propose a generalized plasmon model which allows to follow the development of nanostructures period during diamond irradiation by femtosecond laser pulse at $800 \mathrm{~nm}$ wavelength. We consider the air as the dielectric environment.

We use the plasmon model in the case of dielectric thanks to the specific advantage of femtosecond laser wich allow to create, during its interaction with material, a thin layer that has a metallic character (it will be noted for the following pseudo-metal layer).

The surface plasmon polariton dispersion relation SPP is [12]:

$$
\omega^{2}=c^{2} K_{s p}^{2} \frac{\left(\varepsilon_{p m}+\varepsilon_{d}\right)}{\varepsilon_{p m} \varepsilon_{d}}
$$

Where $\varepsilon_{p m}$ is the dielectric function of the pseudometal layer, $\varepsilon_{d}$ is the dielectric constant of the surrounding the pseudo-metallic layer $\left(\varepsilon_{\text {air }}=1\right.$ and $\varepsilon_{\text {diamond }}=5.7$ at $\left.800 \mathrm{~nm}[10]\right)$.

$K_{s p}=K_{s p 1}+i K_{s p 2}$ Is the plasmon propagation number, $K_{s p 1}=\frac{2 \pi}{\lambda_{s p}}=\frac{\pi}{\Lambda}\left(\lambda_{s p}\right.$ is the plasmon wavelengh, and $\Lambda$ is the nanostructure's period induced in the diamond surface).

The dielectric function of the pseudo-metal layer is given through the Drude's model [13]:

$$
\begin{gathered}
\varepsilon_{p m}=\varepsilon_{p m_{1}}+i \varepsilon_{p m_{2}}= \\
1+\left(\varepsilon_{\text {diamond }}-1\right)\left(1-\frac{n_{e h}}{n_{0}}\right)-\frac{\omega_{p}^{2}}{\omega^{2}} \times\left(\frac{1}{\left.1+\frac{i}{\omega \tau}\right)}\right)
\end{gathered}
$$

where $n_{0}$ is the valence band electron concentration supposed equal to $10^{23} \mathrm{~cm}^{-3} \cdot \tau_{e^{-} e^{-}}$is the electronelectron collision time supposed equal to $1 \mathrm{fs}$.

$\omega_{p}=\sqrt{\frac{n_{e h} e^{2}}{\varepsilon_{0} m_{o p t} m_{e}}}$ is the plasma frequency

( $\varepsilon_{0}=8.85 \times 10^{-12}$ F.m and the optical effective mass $\left.m_{\text {opt }}=0.28[13,14]\right)$.

M. Straub et al [11] have determined the grooves's period of $\mathrm{Si}$ after formalizing formulas of $K_{s p_{1}}$ and $K_{s p_{2}}$ via the ejection of the critical density of plasmon excitation $n_{c r}$ for the objective to simplify the calculation such as $\varepsilon_{p m_{1}}\left(n_{c r}\right)=0$ ( in our case $n_{c r}=3.210^{21} \mathrm{~cm}^{-3}$ at $\lambda=800 \mathrm{~nm}$ ), so this formulas become special case just for the property of Si and the wavelength used.

In our case, we will try in this paper to build a general formula of $K_{s p_{1}}$ and $K_{s p_{2}}$ valid for all semiconductor material and dielectrics and for all femtosecond laser wavelengths.

In the one hand, according to the formula (1)

$$
K_{s p}^{2}=\left(\frac{\omega}{c}\right)^{2} \times\left(\frac{\varepsilon_{p m} \varepsilon_{d}}{\varepsilon_{p m}+\varepsilon_{d}}\right)
$$

In the other hand, we have

$$
\begin{aligned}
& K_{s p}^{2}=\left(K_{s p_{1}}+i K_{s p_{2}}\right)^{2}=K_{s p_{1}}^{2}-K_{s p_{2}}^{2}+2 i K_{s p_{1}} K_{s p_{2}} \\
& \frac{1}{K_{s p}^{2}}=\frac{c^{2}}{\omega^{2}}\left(\frac{1}{\varepsilon_{d}}+\frac{1}{\varepsilon_{p m}}\right)=\frac{c^{2}}{\omega^{2}}\left(\frac{1}{\varepsilon_{d}}+\frac{1}{\left(\varepsilon_{p m_{1}}+i \varepsilon_{p m_{2}}\right)}\right) \\
& \frac{1}{K_{s p}^{2}}=\frac{c^{2}}{\omega^{2}}\left(\frac{\varepsilon_{p m_{1}}^{2}+\varepsilon_{p m_{2}}^{2}+\varepsilon_{d} \varepsilon_{p m_{1}}-i \varepsilon_{d} \varepsilon_{p m_{2}}}{\varepsilon_{d}\left(\varepsilon_{p m_{1}}^{2}+\varepsilon_{p m_{2}}^{2}\right)}\right)
\end{aligned}
$$

We put:

$\alpha=\varepsilon_{p m_{1}}^{2}+\varepsilon_{p m_{2}}^{2}+\varepsilon_{d} \varepsilon_{p m_{1}}$

$\beta=\varepsilon_{d} \varepsilon_{p m_{2}}$

$\gamma=\varepsilon_{d}\left(\varepsilon_{p m_{1}}^{2}+\varepsilon_{p m_{1}}^{2}\right)$

Which implies:

$$
K_{s p}^{2}=\frac{\omega^{2}}{c^{2}}\left(\frac{\gamma}{\alpha-i \beta}\right)
$$

We have also:

$$
\begin{aligned}
& K_{s p_{1}}^{2}-K_{s p_{2}}^{2}=\left(\frac{\omega^{2}}{c^{2}}\right)\left(\frac{\gamma \alpha}{\alpha^{2}+\beta^{2}}\right) \\
& 2 \mathrm{~K}_{s p_{1}} K_{s p_{2}}=\left(\frac{\omega^{2}}{c^{2}}\right)\left(\frac{\gamma \beta}{\alpha^{2}+\beta^{2}}\right)
\end{aligned}
$$

Equation (4) implies $K_{s p_{2}}^{2}=\left(\frac{\omega^{2}}{2 c^{2} K_{s p_{1}}}\right)^{2} \frac{\gamma^{2} \beta^{2}}{\left(\alpha^{2}+\beta^{2}\right)^{2}}$

Replacing in (3), we found:

$$
4 \mathrm{~K}_{s p_{1}}^{4}-4 \frac{\omega^{2}}{c^{2}} \frac{\gamma \alpha}{\left(\alpha^{2}+\beta^{2}\right)} K_{s p_{1}}^{2}-\left(\frac{\omega^{2}}{c^{2}}\right)^{2} \frac{\gamma^{2} \beta^{2}}{\left(\alpha^{2}+\beta^{2}\right)^{2}}=0
$$


To find the roots of this polynomial, we put $\quad x=K_{s p}^{2}$ and :

$$
a=4, \quad b=-4 \frac{\omega^{2}}{c 2} \frac{\gamma \alpha}{\alpha^{2}+\beta^{2}}, d=-\left(\frac{\omega^{2}}{c^{2}}\right)^{2} \frac{\gamma^{2} \beta^{2}}{\left(\alpha^{2}+\beta^{2}\right)^{2}}
$$

then we can write (5) under the form: $a x^{2}+b x+d=0$

We must seek a real and positive value of $K_{s p_{1}}$ which is physically accepted. We finally obtain

$$
\begin{gathered}
K_{s p_{1}}=\frac{1}{\sqrt{2}} \frac{\omega}{c} \sqrt{\frac{\gamma \alpha}{\alpha^{2}+\beta^{2}}+\frac{\gamma}{\sqrt{\alpha^{2}+\beta^{2}}}} \\
K_{s p_{2}}=\frac{\omega^{2}}{2 c^{2} K_{s p_{1}}\left(\alpha^{2}+\beta^{2}\right)}
\end{gathered}
$$

\section{RESULTS AND DISCUSSION:}

When the diamond surface is excited by multipulse laser femtosecon, a fine pseudo-metal layer is build [19] due to the non-thermal fusion (disorder) which takes place at sub-vibrational duration time [15]. It is a special property of femtosecond laser because the pulse duration is less than the electron-phonon relaxation time $[16,17$, 18]. A change of states appears clearly in the optical properties of diamond such reflectivity $R=\frac{(n-1)^{2}+\kappa^{2}}{(n+1)^{2}+\kappa^{2}}$ (refraction index $n=\frac{1}{\sqrt{2}} \sqrt{\varepsilon_{p m_{1}}+\sqrt{\varepsilon_{p m_{1}}^{2}+\varepsilon_{p m_{2}}^{2}}}$ and the absorption index $\left.K=\frac{\varepsilon m_{p m_{2}}}{2 \mathrm{n}}[20]\right)$.

Fig. 1 shows the evolution of the diamond reflectivity in function of density ratio at $\lambda=800 \mathrm{~nm}$. We notice that the reflectivity increase when the plasma density increase $n_{e h}$.

So, after a multipulse femtosecond laser, the diamond take the characteristic of a metal with high free electron density.

When the pulse number increase, the excited electron density $n_{e h}$ increase too until the reflectivity reached $33 \%$ (fig. 1) which correspond to $\varepsilon_{p m}=-\varepsilon_{\text {air }}$, this is the point where the first order nanostructure can be observed with a period $\Lambda_{\min }=397 \mathrm{~nm}$ at $\lambda=800 \mathrm{~nm}$, we will call it minimum plasmon resonance $R P_{\min }$. This $R P_{\min }$ is caused when the plasmon tends to a coherent coupling with the incident laser at air/pseudometallic interface as if the original material (diamond) does not exist.

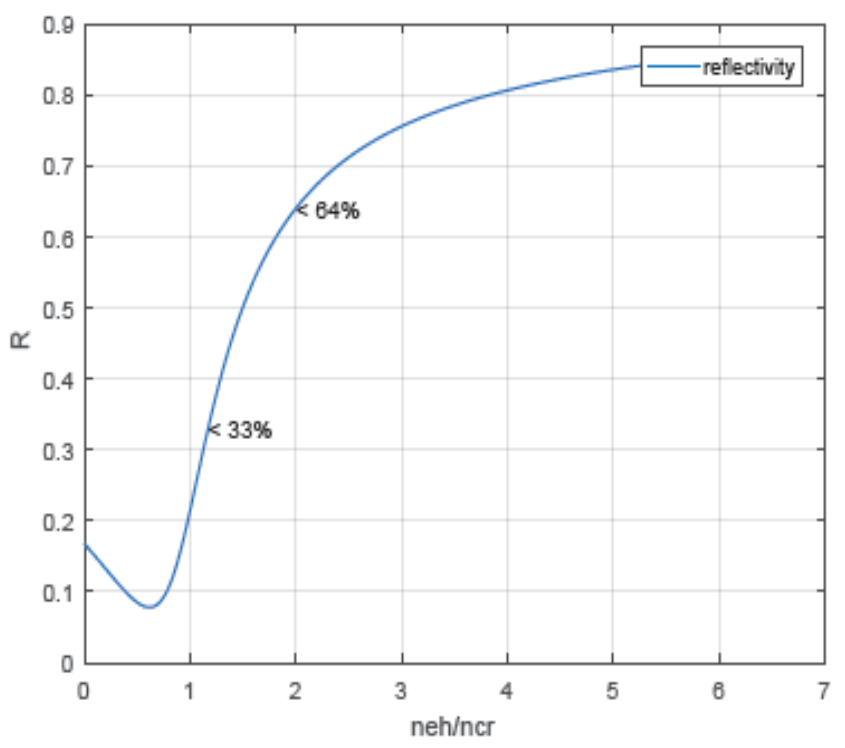

Fig. 1. evolution of the diamond reflectivity in function of density ratio at $\lambda=800 \mathrm{~nm}$. $33 \%$ and $64 \%$ correspond to $R P_{\min }$ and $R P_{\text {max }}$ respectively.

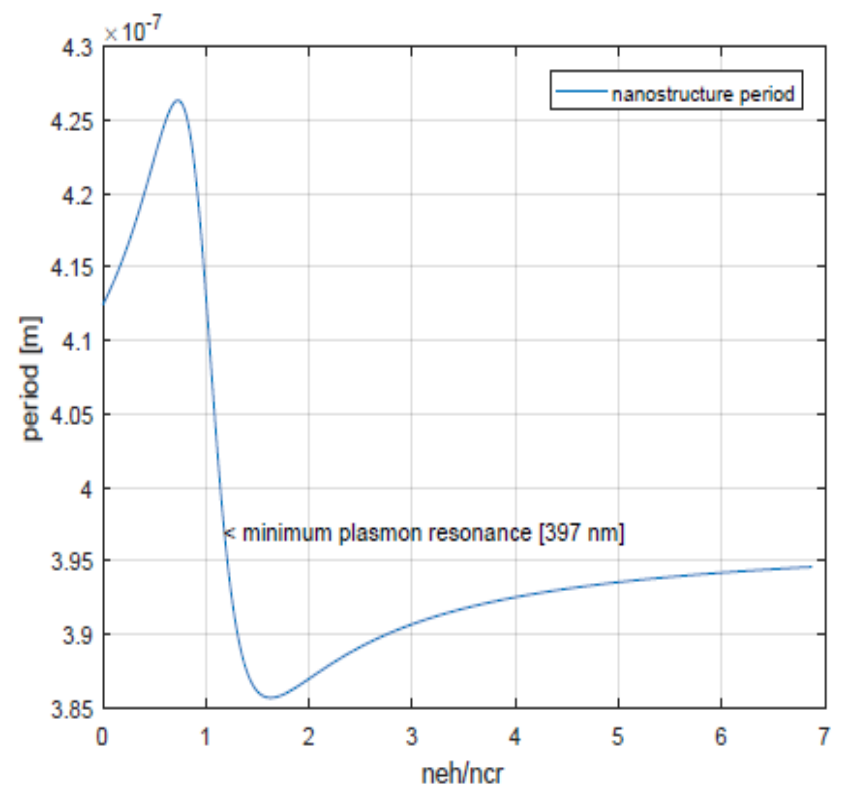

Fig. 2. behaviour of the nanostructure's period $\Lambda$ in function of the density ratio at the interface air/pseudometallic at $\lambda=800 \mathrm{~nm}$

When the number of pulse increase again, the pseudometallic dielectric function decreased until reach $\varepsilon_{p m}=-\varepsilon_{\text {dimond }}$. At this point we find the smallest nanostructure period, also called as the saturation period [2], $\Lambda_{\max }=160 \mathrm{~nm}$ at $\lambda=800 \mathrm{~nm}$ ( fig. 3), due to the maximum plasmon resonance $R P_{\max }$ at the interface pseudo-metal/diamond. 


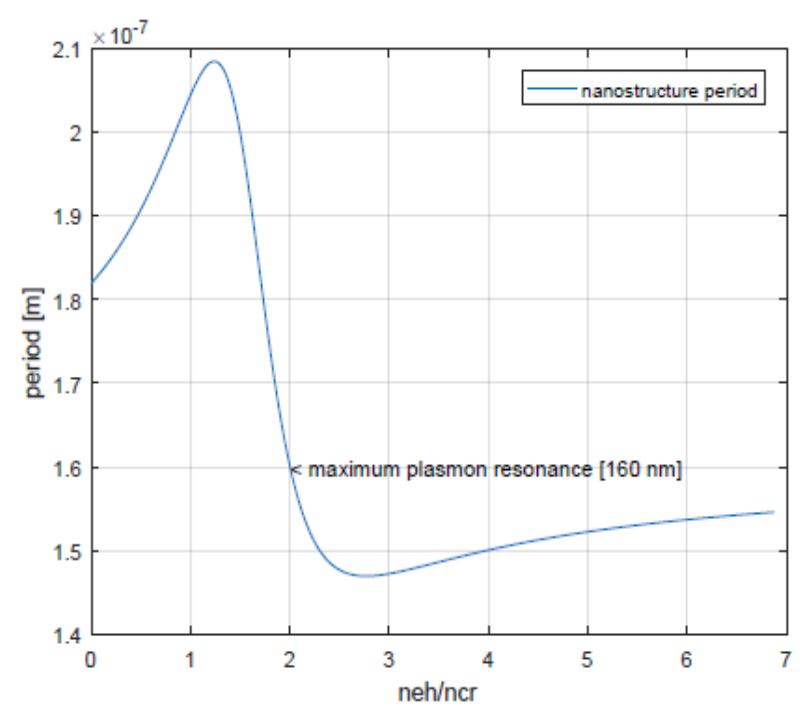

Fig. 3. behaviour of the nanostructure's period $\Lambda$ in function of the density ratio at the interface pseudometallic/diamond at $\lambda=800 \mathrm{~nm}$.

We can conclude that there is like a transverse migration of the surface plasmon polariton SPP form the air/pseudo-metal interface to pseudo-metal/diamond during the increase of femtosecond laser pulse number and the nanostructure period decrease form $\Lambda_{\min }$ to $\Lambda_{\max }$. So we deduce that the ordered nanostructures can be observed only in the range $\left[R P_{\text {min }}, R P_{\text {max }}\right]$.

\section{Conclusion:}

In this paper we have derived a generalized relations of $K_{s p_{1}}$ and $K_{s p_{2}}$ which can be used directly to calculate the period of nanostructures and other optical parameters at any wavelength femtosecond laser incident without need to return to basic relations (1) and (2) for all the dielectric or semiconductor material.

We have used this model to calculate the periods of nanostructures that can be observed on the diamond during irradiation by multipulse femtosecond laser at $800 \mathrm{~nm}$ in air room. We have determined the saturation period $\Lambda_{\max }$ that corresponds to the maximum plasmon resonance $R P_{\max }$. We conclude that the periodic nanostructures can be observed only in the range $\left[R P_{\min }, R P_{\max }\right]$.

\section{References}

[1] T. Q. Jia, H. X. Chen, M. Huang, F. L. Zhao, J. R. Qiu, R. X. Li, Z. Z. Xu, X. K. He, J. Zhang, and H. Kuroda, , Phys. Rev. B 72, 125429 (2005).

[2] S. Richter, M. Heinrich, S. Döring, A. Tünnermann and S. Nolte, Appl Phys A 104, 503 (2011).

[3] M. Huang, F. Zhao, Y. Cheng, N. Xu, and Z. Xu, ACS Nano 3, 12 (2009).

[4] T.H.R. Crawford and H.K. Haugen, App. Sur. Sci. 253, 4970 (2007).

[5] T. Chen, H. Yang, E. Zhao, J. Qian, J. Hao, J. Han, W. Tang and H. Zhu, J. of Russ. Las. Res, 35, 6, (2014)

[6] A. Y. Vorobyev and C. Guo, Las. Phot. Rev. 17, 1 (2012)

[7] S. He, J. J. Nivas, K.K. Anoop, A. Vecchione, M. Hu, R. Bruzzese and S. Amoruso, App. Sur. Sci. 353, 1214 (2015)

[8] G. Miyaji and K. Miyazaki, App. Phys. Lett. 103, 071910 (2013)

[9] K. Miyazaki , G. Miyaji, Phys. Proc. 39, 674 ( 2012 ) [10] M. Shinoda, R. Gattass, and E. Mazur, J. App. Phys. 105, 053102 (2009)

[11] M. Straub, M. Afshar, D. Feili, H. Seidel, and K. Konig, J. App. Phys. 111, 124315 (2012)

[12] Near-field optics and surface plasmon polaritions, edited by S. Kawata (Springer, Berlin, 2001), p. 19.

[13] K. Sokolowski-Tinten and D. von der Linde, Phys. Rev. B 61, 4( 2000)

[14] Diamond Electronic Properties and Applications, edited by Lawrence S. Pan and Don R. Kania, (Springer, New York, 1995), p. 251, 252

[15] G. Sciaini, M. Harb, S. G. Kruglik, T. Payer, C.T. Hebeisen, F.J. Meyer zu Heringdorf, M. Yamaguchi, M. Horn-von Hoegen, R. Ernstorfer and R. J. Dwayne Miller, Nature 458|5, 07788 (2009).

[16] S. K. SUNDARAM and E. MAZUR, Nature mat. 1, 1 ( 2002).

[17] C. V. Shank, R. Yen, and C. Hirlimann, Phys. Rev. Lett. 50, 6 (1983).

[18] T. Zier, Eeuwe S. Zijlstra, A. Kalitsov, I. Theodonis, and M.E. Garci, J. Las. Micro / Nanoengineering 2, 2 ( 2007).

[20] Handbook of Optical Constants of Solids, edited by EDWARD D. PALIK, (Academic Press, USA, 1985), p. 192. 